\title{
Current applications and safety profile of laser interstitial thermal therapy in the pediatric population: a systematic review of the literature
}

\author{
Sabrina Zeller, MD, ${ }^{1}$ Joel Kaye, MD, ${ }^{1}$ Fareed Jumah, MD, ${ }^{1}$ Shilpa S. Mantri, BS, BA, ${ }^{2}$ \\ Jamshaid Mir, BA, ${ }^{3}$ Bharath Raju, MD, ${ }^{1}$ and Shabbar F. Danish, MD ${ }^{1}$ \\ 'Department of Neurosurgery, Rutgers-Robert Wood Johnson Medical School and Robert Wood Johnson University Hospital, \\ New Brunswick; ${ }^{2}$ Rutgers-Robert Wood Johnson Medical School, New Brunswick, New Jersey; and ${ }^{3}$ College of Medicine, \\ Northeast Ohio Medical University, Rootstown, Ohio
}

\begin{abstract}
OBJECTIVE Laser interstitial thermal therapy (LITT) provides a minimally invasive alternative to open brain surgery, making it a powerful neurosurgical tool especially in pediatric patients. This systematic review aimed to highlight the indications and complications of LITT in the pediatric population.

METHODS In line with the PRISMA guidelines, the authors conducted a systematic review to summarize the current applications and safety profiles of LITT in pediatrics. PubMed and Embase were searched for studies that reported the outcomes of LITT in patients < 21 years of age. Retrospective studies, case series, and case reports were included. Two authors independently screened the articles by title and abstract followed by full text. Relevant variables were extracted from studies that met final eligibility, and results were pooled using descriptive statistics.

RESULTS The selection process captured 303 pediatric LITT procedures across 35 studies. Males comprised approximately $60 \%$ of the aggregate sample, with a mean age of 10.5 years (range $0.5-21$ years). The LITT technologies used included Visualase (89\%), NeuroBlate (9\%), and Multilase $2100(2 \%)$. The most common indication was treatment of seizures $(86 \%)$, followed by brain tumors $(16 \%)$. The mean follow-up duration was 15.6 months (range 1.3-48 months). The overall complication rate was $15.8 \%$, which comprised transient neurological deficits, cognitive and electrolyte disturbances, hemorrhage, edema, and hydrocephalus. No deaths were reported.
\end{abstract}

CONCLUSIONS As of now, LITT's most common applications in pediatrics are focused on treating medically refractory epilepsy and brain tumors that can be difficult to resect. The safety of LITT can provide an attractive alternative to open brain surgery in the pediatric population.

https://thejns.org/doi/abs/10.3171/2021.2.PEDS20721

KEYWORDS laser interstitial thermal therapy; pediatrics; indications; complications; systematic review; surgical technique

T ASER interstitial thermal therapy (LITT) is a minimally invasive procedure that uses the nonionizing radiation of laser light to produce thermal effects on tissue, resulting in cell death. With advancements in laser and imaging technology, LITT has become increasingly popular in the treatment of intracranial pathologies, including pediatric neuro-oncology and epilepsy. ${ }^{1}$ Although LITT was first used to treat brain tumors in the 1990s, the first reported use of LITT in a pediatric brain tumor only dates back to 2011, with the treatment of a supratentorial primitive neuroectodermal tumor in a 10 -year-old child. ${ }^{1-4}$ Since then, this modality has been used to treat various other pediatric tumor-specific pathologies, including sub- ependymal giant cell astrocytoma, pilocytic astrocytoma, ependymoma, medulloblastoma, choroid plexus xanthogranuloma, and ganglioglioma, in addition to epileptogenic foci, such as hypothalamic hamartomas (HHs) and cortical tubers..$^{5-7}$ LITT offers advantages in the treatment of pediatric patients via its minimally invasive nature, with shortened hospital stays and decreased radiotherapy or chemotherapy requirements that minimize the psychosocial impact on children's lives. ${ }^{6,8}$

Although LITT is a minimally invasive procedure, complications have been reported in the literature. These include hemorrhage, infection, neurological deficits, edema, and technical issues, which are also classically seen 


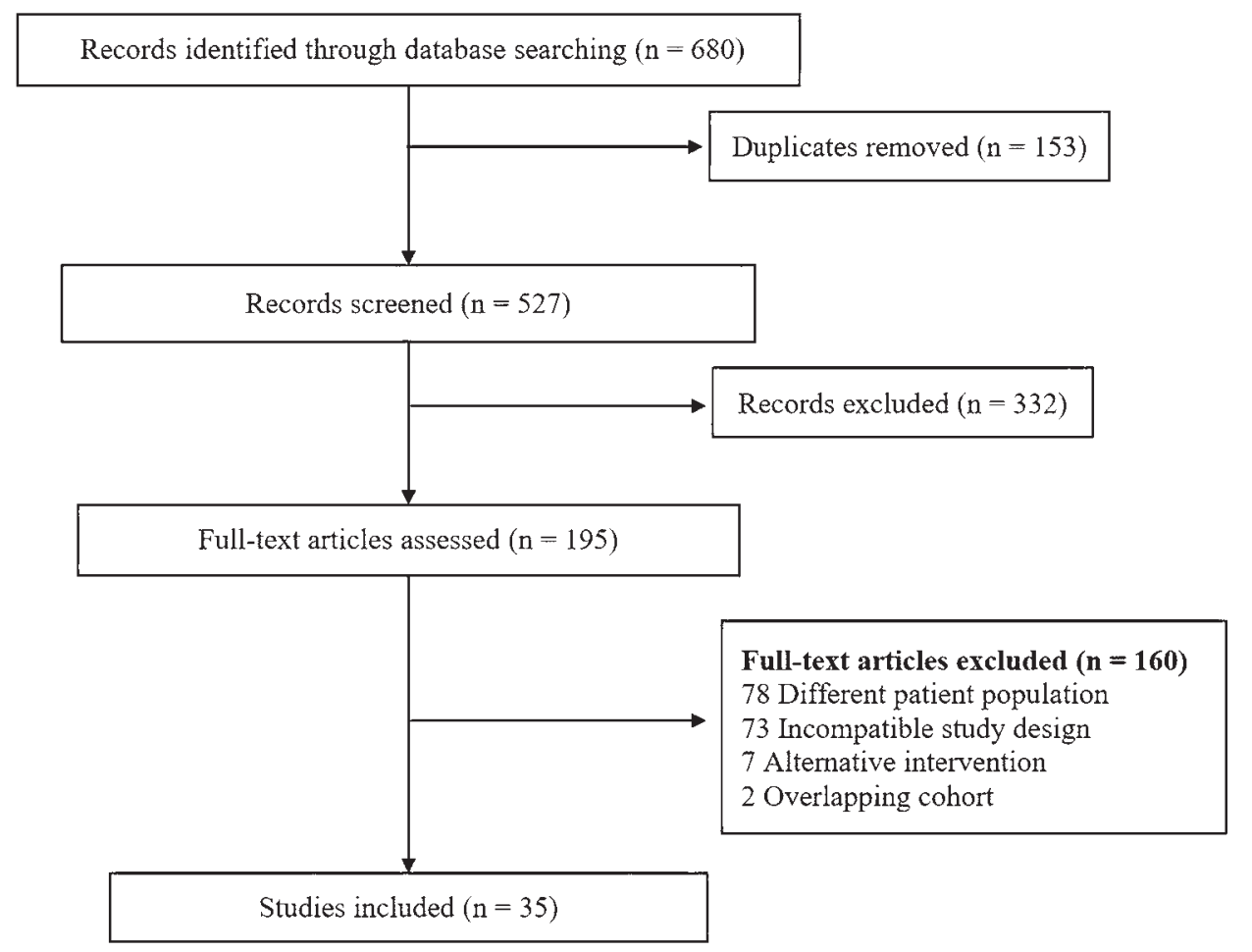

FIG. 1. PRISMA flow diagram that shows the screening and selection process used for this systematic review of LITT in the pediatric population.

with other neurosurgical procedures. ${ }^{1}$ Due to its relatively novel use, there have not been high-powered studies investigating its impact in the pediatric population. ${ }^{9}$ In an attempt to provide insight into the utility of LITT in the pediatric population, we systematically reviewed the literature to summarize the current applications and the safety profile of LITT in the treatment of pediatric intracranial pathologies.

\section{Methods}

This systematic review was conducted in line with the Preferred Reporting Items for Systematic Reviews and Meta-Analyses (PRISMA) guidelines. ${ }^{10}$ Ethical approval and patient consent were not required for this study.

\section{Search Strategy and Selection Criteria}

A comprehensive literature search, last updated on February 20, 2020, was performed in PubMed and Embase using the search string ("laser interstitial thermal therapy" OR "laser interstitial thermotherapy" OR "laser thermal ablation" OR LITT) AND neurosurg*. After removal of duplicates, the articles were screened based on the following criteria: 1) patients $<21$ years of age; 2) treated with LITT; 3) articles reporting complications associated with LITT; and 4) randomized controlled trials, cohort studies, case series, and case reports. Non-English articles, animal studies, reviews, and studies lacking the desired outcomes were excluded. Using Covidence, two authors (J.M., S.S.M.) independently carried out screening of the titles and abstracts, followed by full-text screening of the resultant articles. Conflicts were resolved by discussion with a third author (F.J.).

\section{Data Extraction and Statistical Analysis}

From the articles that met final eligibility, two authors (F.J., S.Z.) extracted the following parameters: author, year, study design, sample size, age, sex, LITT technology, indication for treatment, histopathological diagnosis, lesion location, pretreatment lesion volume and average maximum diameter, treatment time, follow-up duration, and complications. Pooled variables were expressed in percentages, means, standard deviations, and ranges by using Microsoft Excel for Office 365 version 1912.

\section{Results}

\section{Search Outcome}

The primary search identified 680 titles (Fig. 1). After 153 duplicates were removed, title and abstract screening yielded 195 candidate articles. The systematic full-text assessment resulted in 35 articles for final inclusion, which included 1 (3\%) prospective cohort study, ${ }_{11}^{11} 13$ (37\%) retrospective cohort studies, ${ }^{4,9,12-22} 9(26 \%)$ case reports, ${ }^{2,3}$, ${ }^{23-29} 10(29 \%)$ case series, ${ }^{5-7,30-36}$ and $2(6 \%)$ conference

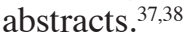

\section{Demographic Data and Clinical Features}

The included articles contained a total of 409 patients; however, for the purposes of our analysis we excluded 98 patients for age $>21$ years, 8 patients from a repeated co- 
hort, and 7 patients who underwent a procedure other than LITT. This left us with 296 patients in the systematic review, 7 of whom underwent a repeat LITT procedure, for a total of 303 LITT cases.

Males constituted 59\% (151/257) of the aggregate patient sample, with a mean age of 10.5 years (range $0.5-21$ years). The most common indications for LITT were epileptogenic foci, particularly HHs $(n=161)$, focal cortical dysplasia $(n=31)$, and tuberous sclerosis cortical tubers ( $\mathrm{n}$ $=23$ ), along with deep-seated and/or progressing tumors $(n=47)$ (Table 1). The mean follow-up duration was 15.6 months (range 1.3-48 months). In terms of pathology, there were an additional 7 patients with tumors $(n=47)$ due to overlap between the epilepsy indication group with symptomatic tumor progression. The remaining 2 patients underwent LITT for the treatment of symptomatic radiation necrosis and behavioral symptoms associated with an $\mathrm{HH}$.

The locations that underwent LITT were recorded in $78 \%$ of the total number of cases $(n=235)$. Of the 235 , the most common locations included the hypothalamus (68\%), temporal lobe $(8 \%)$, frontal lobe $(7 \%)$, ventricles $(6 \%)$, and thalamus (4\%). Other, less common locations consisted of the occipital lobe, parietal lobe, brainstem, cerebellum, caudate, tentorium, and optic nerve.

Multiple LITT technologies were used; Visualase Thermal Therapy System (Visualase, Inc.) was used in the majority of cases (188/211, 89\%); Monteris Medical NeuroBlate System (Monteris Medical, Inc.) in 9\% (18/211) of cases; and Multilase 2100 (Technomed International, Inc.) in $2 \%(4 / 211)$. One patient was treated using an unspecified Nd:YAG 1.06-mm laser.

\section{Complications}

A total of 55 complications were observed in 48 $(15.8 \%)$ procedures (Table 2). Of these complications, 5 were insertion related, 46 were ablation related, and the remaining 4 were unspecified.

Focal neurological deficits occurred in $9(3.0 \%)$ cases and consisted of both motor and sensory deficits (paresis, visual field cuts, and Horner's syndrome). Cognitive disturbances involving memory, language, or behavioral changes were also seen in 9 (3.0\%) cases. Four (1.3\%) patients experienced worsened seizures; 1 progressed to status epilepticus with respiratory failure but went on to make a full recovery. Of these neurological complications, $17 / 22(77.3 \%)$ were transient deficits.

On the other hand, 8 (2.6\%) patients experienced metabolic and electrolyte disturbances. Five patients had disturbances in sodium metabolism, and 3 patients reported weight gain or hypothyroidism. It should be noted that $7 / 8$ of these patients were being treated for HHs.

Hemorrhage was seen in 4 (1.3\%) patients, without persistent deficits; whereas 2 patients did not require further intervention, 1 patient with a twist drill-related epidural hematoma formation had an evacuation without further sequelae, and the remaining patient experienced improper catheter placement resulting in subarachnoid hemorrhage/ intraventricular hemorrhage with hydrocephalus, which resolved after placement of a temporary lumbar drain.

Symptomatic edema was explicitly reported as a complication in 2 patients. One patient was noted to have associated postoperative somnolence and hemiparesis; however, the edema progressively decreased over time. The second patient's edema was further complicated by Decadron-induced gastritis. The apparent lack of edema reported as a complication is addressed further in the discussion. Four cases of hydrocephalus were reported, along with 1 case of shunt obstruction.

Ten of the complications were due to technical difficulties associated with the LITT procedure. There were 5 cases with incorrect catheter placement; whereas 3 of these misplacements resulted in decreased efficacy of the LITT procedure without further complication, 1 required that the procedure be aborted, followed by open resection, and 1 resulted in subarachnoid hemorrhage/intraventricular hemorrhage, as described above. A single patient experienced device malfunction in which the cooling mechanism failed, resulting in overheating and breakage of the laser tip; the laser tip was left in situ following partial ablation, and the patient experienced no further sequelae. Four other patients received incomplete treatments secondary to unspecified technical difficulties.

Four of the complications were noted as unspecified minor complications. Of note, there was no mortality or infection reported as a result of LITT in the pediatric cohort.

\section{Discussion \\ Benefits of LITT}

LITT is a novel and minimally invasive procedure that has transformed the treatment of various intracranial pathologies, leading to its widespread adaptation as a neurosurgical treatment alternative. ${ }^{1}$ Current LITT systems allow surgeons to selectively ablate tumors and lesions in the brain-including malignant gliomas, radiosurgery-resistant metastases, lesions of epileptogenic foci, and radiation necrosis - that may have previously been considered inoperable, difficult to access, or unsafe to resect based on their location in or near areas of eloquence. ${ }^{39,40}$

The minimally invasive nature of LITT offers important advantages over open procedures, especially among pediatric populations. Postoperatively, not only has the procedure shown a lower risk of infection but also it has been associated with significantly shortened recovery time and duration of hospitalization..$^{11,41}$ Decreasing hospitalization time is of importance in minimizing psychological impact and disruption to a child's life. ${ }^{1}$ Due to its lack of ionizing radiation exposure, LITT can be repeated in cases of progression. ${ }^{41}$ LITT may also decrease the need for other forms of treatment, such as radiotherapy or chemotherapy, thereby eliminating other potential sources of stress. ${ }^{1}$ Requiring only a single stab incision and stitch, the procedure decreases the risk of wound complications and avoids hair shaving, thus minimizing cosmetic changes in children., ${ }^{1,42}$

Furthermore, LITT enables real-time visualization of the ablation with MRI thermometry, discrete lesion margins, and immediate efficacy and control during laser energy delivery. ${ }^{11}$ The instantaneous feedback allows for immediate confirmation of the stereotactic trajectory, tight control of the ablation volume, and operation in deep parts of the brain with accurate estimates of thermal damage. ${ }^{11,39}$ 
TABLE 1. Reported indications and complications for LITT in pediatric patients

\begin{tabular}{|c|c|c|c|}
\hline $\begin{array}{l}\text { Authors } \\
\& \text { Year }\end{array}$ & $\begin{array}{c}\text { No. of } \\
\text { Patients }\end{array}$ & $\begin{array}{l}\text { Pathological } \\
\text { Diagnosis (no., \%) }\end{array}$ & $\begin{array}{c}\text { No. of Ops w/ } \\
\text { Complications } \\
(\%)\end{array}$ \\
\hline Ascher, $1990^{2}$ & 1 & Astrocytoma grade $2(1,100 \%)$ & $0(0 \%)$ \\
\hline Boerwinkle et al., $2018^{12}$ & 51 & $\mathrm{HH}(51,100 \%)$ & $0(0 \%)$ \\
\hline Miller et al., $2017^{19}$ & 10 & $\begin{array}{l}\mathrm{FCD}(3,30 \%) ; \text { LGG }(2,20 \%) ; \text { SEGA }(1,10 \%) ; \text { JPA }(1,10 \%) ; \text { immature teratoma }(1,10 \%) ; \\
\text { oligodendroglioma (1, 10\%); NR (1, 10\%) }\end{array}$ & $0(0 \%)$ \\
\hline Buckley et al., $2016^{13}$ & 10 & HH (4, 40\%); SEGA (3, 30\%); optic glioma (1, 10\%); ganglioglioma (1, 10\%); PXA (1, 10\%) & $4(40 \%)$ \\
\hline Cobourn et al., $2018^{14}$ & 4 & $\mathrm{FCD}(2,50 \%) ; \mathrm{TSC}(2,50 \%)$ & $0(0 \%)$ \\
\hline Dadey et al., $2016^{5}$ & 2 & $\operatorname{SEGA}(2,100 \%)$ & $0(0 \%)$ \\
\hline Dadey et al., $2016^{30}$ & 2 & MTS (1,50\%); mesiotemporal cystic lesion (1,50\%) & $0(0 \%)$ \\
\hline Donos et al., $2018^{15}$ & 5 & MTS (1, 20\%); NR, w/o MTS (4, 80\%) & $0(0 \%)$ \\
\hline Du et al., $2017^{16}$ & 4 & $\mathrm{HH}(4,100 \%)$ & $0(0 \%)$ \\
\hline Fayed et al., $2018^{9}$ & 11 & $\mathrm{HH}(4,36.4 \%) ;$ periventricular heterotopia (3, 27.3\%); TSC (2, 18.2\%); FCD (2, 18.2\%) & $0(0 \%)$ \\
\hline Feroze et al., $2020^{23}$ & 2 & Atypical teratoid/rhabdoid tumor (2, 100\%) & $0(0 \%)$ \\
\hline Gadgil et al., $2020^{17}$ & 58 & $\mathrm{HH}(58,100 \%)$ & $14(24.1 \%)$ \\
\hline Sandoval-Garcia et al., $2018^{37}$ & 39 & $\begin{array}{l}\text { Hippocampal sclerosis }(6,15.4 \%) ; \mathrm{HH}(3,7.7 \%) ; \operatorname{TSC}(4,11.3 \%) ; \mathrm{FCD}(11,28.2 \%) ; \text { postre- } \\
\text { section residual target }(14,35.9 \%) ; \mathrm{NR}(1,2.6 \%)\end{array}$ & $8(20.5 \%)$ \\
\hline Gonzalez-Martinez et al., $2014^{24}$ & 1 & Periventricular heterotopia $(1,100 \%)$ & $0(0 \%)$ \\
\hline Hooten et al., $2018^{25}$ & 1 & TSC $(1,100 \%)$ & $0(0 \%)$ \\
\hline Jethwa et al., $2011^{3}$ & 1 & Supratentorial PNET (1, 100\%) & $0(0 \%)$ \\
\hline Jethwa et al., $2012^{31}$ & 2 & Supratentorial PNET (1,50\%); ependymoma (1,50\%) & $1(50 \%)$ \\
\hline Kang et al., $2016^{11}$ & 4 & MTS $(4,100 \%)$ & $0(0 \%)$ \\
\hline Karsy et al., $2018^{26}$ & 2 & $\operatorname{SEGA}(2,100 \%)$ & $1(50 \%)$ \\
\hline Kuo et al., $2019^{4}$ & 5 & Ganglioglioma (2, 40\%); radiation necrosis (1, 20\%); gliosis (1, 20\%); FCD (1, 20\%) & $1(20 \%)$ \\
\hline Lewis et al., $2015^{18}$ & 19 & $\begin{array}{l}\text { FCD (10, 52.6\%); TSC (4, 21.1\%); ganglioglioma (1, 5.3\%); ganglioma (1, 5.3\%); PXA (1, } \\
\text { 5.3\%); HH (1, 5.3\%); Rasmussen's encephalitis (1, 5.3\%) }\end{array}$ & $4(21.1 \%)$ \\
\hline Lopez Bon et al., $2014^{38}$ & 6 & DNET (1, 16.7\%); periventricular heterotopia (1, 16.7\%); FCD (2, 33.3\%); HH (2, 33.3\%) & $5(83.3 \%)$ \\
\hline $\begin{array}{l}\text { Riordan \& Tovar-Spinoza, } \\
2014^{29}\end{array}$ & 1 & Ependymoma $(1,100 \%)$ & $0(0 \%)$ \\
\hline Roux et al., $1992^{32}$ & 4 & Pilocytic astrocytoma (2, 50\%); fibrillar astrocytoma (1, 25\%); oligodendrocytoma (1, 25\%) & $0(0 \%)$ \\
\hline Schroeder et al., $2014^{20}$ & 1 & Anaplastic astrocytoma $(1,100 \%)$ & $1(100 \%)$ \\
\hline Southwell et al., $2018^{33}$ & 5 & $\mathrm{HH}(5,100 \%)$ & $1(20 \%)$ \\
\hline Stellon et al., $2019^{34}$ & 3 & TSC $(3,100 \%)$ & $0(0 \%)$ \\
\hline Tandon et al., $2018^{35}$ & 1 & $\mathrm{HH}(1,100 \%)$ & $0(0 \%)$ \\
\hline Tovar-Spinoza et al., $2013^{27}$ & 1 & $\mathrm{HH}(1,100 \%)$ & $0(0 \%)$ \\
\hline Tovar-Spinoza \& Choi, $2016^{7}$ & 11 & $\begin{array}{l}\text { Pilocytic astrocytoma }(6,54.5 \%) \text {; SEGA }(1,9.1 \%) \text {; ependymoma }(1,9.1 \%) \text {; medulloblas- } \\
\text { toma }(1,9.1 \%) \text {; choroid plexus xanthogranuloma }(1,9.1 \%) ; \text { ganglioglioma }(1,9.1 \%)\end{array}$ & $2(18.2 \%)$ \\
\hline Tovar-Spinoza \& Choi, $2016^{6}$ & 1 & Ganglioglioma $(1,100 \%)$ & $0(0 \%)$ \\
\hline Tovar-Spinoza et al., $2018^{21}$ & 7 & TSC $(7,100 \%)$ & $0(0 \%)$ \\
\hline Wilfong \& Curry, $2013^{36}$ & 15 & $\mathrm{HH}(15,100 \%)$ & $1(6.7 \%)$ \\
\hline Xu et al., $2015^{28}$ & 1 & Pilocytic astrocytoma (1, 100\%) & $0(0 \%)$ \\
\hline Xu et al., $2018^{22}$ & 12 & $\mathrm{HH}(12,100 \%)$ & $5(41.7 \%)$ \\
\hline Total & 303 & $\begin{array}{l}\text { HH (161); FCD (31); TSC (23); postresection residual target (14); pilocytic astrocytoma (10); } \\
\text { SEGA (9); ganglioglioma (6); MTS (6); hippocampal sclerosis (6); NR (6); periventricular het- } \\
\text { erotopia (5); ependymoma (3); PXA (2); LGG (2); supratentorial PNET (2); atypical teratoid/ } \\
\text { rhabdoid tumor (2); DNET (1); fibrillar astrocytoma (1); astrocytoma grade } 2 \text { (1); anaplastic } \\
\text { astrocytoma (1); ganglioma (1); oligodendroglioma (1); oligodendrocytoma (1); medulloblas- } \\
\text { toma (1); choroid plexus xanthogranuloma (1); immature teratoma (1); optic glioma (1); radia- } \\
\text { tion necrosis (1); gliosis (1); Rasmussen's encephalitis (1); mesiotemporal cystic lesion (1) }\end{array}$ & $48(15.8 \%)$ \\
\hline
\end{tabular}

DNET = dysembryoplastic neuroepithelial tumor; $F C D=$ focal cortical dysplasia; JPA = juvenile pilocytic astrocytoma; $L G G=$ low-grade glioma; $M T S=$ mesial temporal sclerosis; NR = not reported; PNET = primitive neuroectodermal tumor; PXA = pleomorphic xanthoastrocytoma; $\mathrm{SEGA}=$ subependymal giant cell astrocytoma; TSC = tuberous sclerosis cortical tuber. 


\section{Complications of LITT}

Although LITT is a minimally invasive procedure, complications still occur in the general population, at a rate of $12.9 \%$ in a recent national study, with a mortality rate of $2.5 \% .^{43}$ Neurological deficits are the most commonly reported postoperative complications after LITT, and are often the direct result of LITT hyperthermia and postablation edema. ${ }^{1,44,45}$ In our review there were 9 focal neurological deficits reported, with hemiparesis and visual field cuts being the most common. Additional neurological complications included 9 reports of cognitive disturbances and 4 cases of worsened seizures. The majority of the cognitive disturbances, the most common of which was short-term memory loss, were seen in patients with $\mathrm{HH}$. Short-term memory loss is consistently one of the most common complications after $\mathrm{HH}$ surgery regardless of treatment modality ${ }^{46,47}$ Of the total neurological complications, $77.3 \%$ were transient, which is consistent with literature reports that the adverse events after LITT infrequently result in permanent neurological morbidity. ${ }^{48,49}$

For those complications that were transient in nature, a few articles specified the time to symptom resolution. One case of Horner's syndrome was resolved 3 months after the procedure. ${ }^{22}$ Tovar-Spinoza and Choi reported 1 case of transient hemiparesis, which had reduced into mild right-hand weakness and dysarthria at an 18-month postoperative follow-up? Other studies in this review reported no permanent neurological deficits and did not provide a definitive timeline for resolution of transient neurological symptoms. In comparison, a literature review by Ashraf et al. suggested complete or partial resolution of postoperative motor complications, including paresis, facial droop, and hemiplegia, within 1 month. ${ }^{1}$

Although clinically significant edema was confirmed as an independent complication in only 2 patients, it is likely that postablation edema also played a role in many of the transient postoperative symptoms reported in other patients. ${ }^{1}$ This discrepancy highlights the heterogeneity in reporting and classifying complications among the included studies.

Disturbance of sodium metabolism was another common complication seen among patients in our review. This was identified mostly in patients with $\mathrm{HH}$, but also in 1 patient with an anaplastic ependymoma of the third ventricle. The large number of patients with $\mathrm{HH}$ in this review probably accounts for the high frequency of complications expected with disturbance to the hypothalamus, including memory disturbance, sodium imbalance, weight gain, and Horner's syndrome. ${ }^{50}$

Technical complications accounted for $18.2 \%(n=10)$ of the reported complications in this review. These were a result of suboptimal catheter placement and technical difficulties with the device, which resulted in hemorrhage and ineffective ablation requiring reoperation. Of the 5 catheter misplacements and 1 device malfunction in this review, none of the patients went on to have persistent complications. Nonetheless, minimization of procedural complications will allow for more effective treatment and reduce the necessity of a more invasive procedure, which occurred in 1 patient who required conversion to open resection.

Last, as a known benefit of LITT, the risk of wound
TABLE 2. Frequency and type of complications associated with LITT in pediatric patients

\begin{tabular}{cc}
\hline \multicolumn{1}{c}{ Complication } & No. $(\%)$ \\
\hline Technical complications & $10(18.2 \%)$ \\
\hline Incorrect catheter placement & 5 \\
\hline Device malfunction & 1 \\
\hline Unspecified technical difficulties & 4 \\
\hline Neurological deficits & $9(16.4 \%)$ \\
\hline Motor deficits & 5 \\
\hline Visual field cuts & 3 \\
\hline Horner's syndrome & 1 \\
\hline Cognitive disturbances & $9(16.4 \%)$ \\
\hline Memory loss & 5 \\
\hline Worsened seizures & $4(7.3 \%)$ \\
\hline Status epilepticus w/ respiratory failure & 1 \\
\hline Metabolic \& electrolyte disturbances & $8(14.5 \%)$ \\
\hline Dl/sodium disturbance & 5 \\
\hline Weight gain/hypothyroidism & 3 \\
\hline Hemorrhage & $4(7.3 \%)$ \\
\hline Clinically significant edema & $2(3.6 \%)$ \\
\hline Hydrocephalus/shunt obstruction & $5(9.1 \%)$ \\
\hline Unspecified self-limiting minor complications & $4(7.3 \%)$ \\
\hline Total & 55 \\
\hline DI &
\end{tabular}

$\mathrm{DI}=$ diabetes insipidus

infections is reduced, and notably there were no infections reported in the pediatric population. Furthermore, there was no death in this cohort as a direct complication of the LITT procedure.

\section{Comparison to Alternative Treatment Modalities-Epilepsy}

Overall, $86 \%$ of the patients included in this review underwent LITT for the treatment of epilepsy, with a $15.3 \%$ cumulative rate of adverse events. The most common pathology in patients with epilepsy was $\mathrm{HH}$, which represented $53.1 \%$ of all cases included in the systematic review, followed by focal cortical dysplasia (10.2\%) and tuberous sclerosis cortical tubers (7.6\%). Although a thorough investigation of the efficacy of LITT in the treatment of epilepsy is beyond the scope of this paper, there are some promising outcome data. For example, studies in which the Engel classification was used to evaluate seizure reduction following LITT treatment reported Engel class I outcomes in $74.1 \%$ of cases. ${ }^{9,12,13,16,18,33,35,36}$ Nonetheless, the majority of the remaining studies had variable and subjective definitions of seizure reduction, which hinders our ability to accurately measure the efficacy of LITT in that aspect using all the available data.

Alternative modalities of epilepsy surgery include open resection, endoscopic resection, stereotactic radiosurgery (SRS), and radiofrequency thermocoagulation. Resective surgery, the most described modality in the treatment of pediatric epilepsy, has been reported to have a minor neurological complication rate of $11.2 \%$ and a major neurological complication rate of $5.1 \%$, compared to a total 
neurological complication rate of $7.3 \%-8.8 \%$ in our review. ${ }^{51}$ Neurological deficits vary depending on location, with visual field deficits more prevalent following temporal lobe resection and hemiparesis most prevalent in extratemporal resection..$^{51,52}$ Hader et al. also reported CSF leakage in $14.3 \%$ of patients. ${ }^{51}$ Notably, CSF leakage was not identified as a complication of LITT in any of the studies included in this review.

Specifically in $\mathrm{HH}$, a previous case series describing open resection reported complication rates of $36 \%-51 \%$, which included thalamic infarcts, short-term memory impairment, visual defects, and hydrocephalus. ${ }^{47}$ This reported complication rate is higher than that of LITT, which was $15.2 \%$ for patients with $\mathrm{HH}$ in this review. Additionally, endoscopic resection of $\mathrm{HH}$ has been described previously, with a complication rate of $25 \%$ and a $13.6 \%$ incidence of short-term memory loss; the latter figure is similar to that reported after open surgery. ${ }^{47,53}$ LITT appears to offer advantages over both open and endoscopic resection as far as minimizing memory loss symptoms, with memory disturbance reported in only $5(3.1 \%)$ patients and with persistence in only $1(0.6 \%)$.

Data are limited regarding the use of SRS specifically in pediatric epilepsy, but SRS has been reported as a safe, effective modality in the treatment of pediatric $\mathrm{HH} .{ }^{47}$ The benefits of LITT versus SRS were identified in a metaanalysis examining the management of medically intractable temporal lobe epilepsy among both pediatric and adult patients, with an overall complication rate of $20 \%$ in patients treated with LITT compared to $32 \%$ in those treated with SRS. ${ }^{54}$ The majority of these studies examine SRS in adult patients, and there is a paucity of pediatric studies. In a small study of 27 pediatric patients with $\mathrm{HH}$, Régis et al. reported that there were no neurological deficits following SRS. Whereas this study did not report any neurological complications, there was a $14.8 \%$ rate of transiently increased seizures and a $10 \%$ incidence of transient poikilothermia. ${ }^{55}$ LITT also has a risk of inducing transiently worsened seizures; however, this was seen at a much lower rate, in only $4(2.5 \%)$ patients with $\mathrm{HH}$. It should also be noted that there is a delay to seizure remission with SRS. ${ }^{47,56}$ In spite of these comparisons, LITT and SRS are not meant to be in competition with one another and can in fact be viewed as complementary procedures, each with its own advantages and disadvantages.

Radiofrequency thermocoagulation offers a similar minimally invasive approach to HH as LITT does. In contrast to LITT, a large study consisting mostly of pediatric patients reported frequent complications, the most common of which were transient Horner's syndrome (60.0\%), hyperphagia (27.9\%), and hyponatremia (22.1\%). Memory disturbance was seen in $8.6 \%$ of patients. ${ }^{50}$

\section{Comparison to Alternative Treatment Modalities-Tumor}

Tumor was the second most common indication among the patients included in our review, comprising $15.5 \%$ of the total cases, and yielded a complication rate of $19.1 \%$. This is consistent with complication rates $(8 \%-21.2 \%)$ reported in a review by Banerjee et al., which consisted of mostly adult patients. ${ }^{41}$

Previous studies on resection of pediatric brain tu- mors report overall complication rates up to $69 \%$, with a $12.8 \%-44 \%$ rate of neurological complications. ${ }^{57,58}$ Similar to our review, the most common tumor pathology in these studies was astrocytoma. CSF leakage was one of the most common complications associated with resection (7.3\%). ${ }^{57}$ Importantly, whereas infection was not found as a complication in our review of LITT, Lassen et al. reported a 1.8\% incidence of meningitis and Neervoort et al. reported an overall infection rate of $21 \%$. Other serious complications included severe neurological deficits $(2.9 \%)$, cerebral infarction $(1.5 \%)$, and surgery-related mortality $(0.4 \%-0.8 \%))^{57,58}$

It should be noted that the majority of the cases included in our review were described as "deep seated" or otherwise not safely accessible via typical surgical approaches, which represents a confounding factor when making these comparisons. When reported, tumors were most commonly located in periventricular regions $(34.2 \%)$ or in the thalamus (23.7\%). Although craniotomies offer a comparable rate of adverse events compared to that found in this review, the surgical complexity, and by extension the surgical morbidity, of the cases reported by Lassen et al. is likely to be much lower than would be expected if resection were attempted for the tumors included in the LITT cohort.

The use of SRS for pediatric tumors has been previously reported to have a $16.7 \%-26 \%$ risk of adverse events, along with radiological evidence of edema or radionecrosis. ${ }^{59,60}$ Furthermore, SRS is not typically considered for those $<3$ years old to avoid long-term neurotoxic effects and neurocognitive deficits that may arise because their brains are more susceptible to radiation injury. ${ }^{60}$

\section{Recommendations for Minimizing Complications of LITT}

Minimization of complications can be achieved with strict attention to the pathology and its location. ${ }^{44}$ Hyperthermic damage leading to neurological symptoms may be mitigated by using lower power in target regions that are close to eloquent structures, along with a $3-\mathrm{mm}$ diffusing tip, which allows for greater control of ablation. ${ }^{1}$ Further caution should be taken when the target lesions are not separated from critical areas by CSF or vasculature, because these help dissipate heat. ${ }^{48}$ For larger lesions near critical areas, control of postablation edema is a high priority and can be lessened by using staged procedures or preprocedure initiation of high-dose steroids. 3,61

Additional minimization of procedural complications will come with increasing operator experience. Trajectory planning plays a critical role in the LITT procedure in order to avoid CSF spaces and vasculature. For longer trajectories, accuracy for the target lesion can be improved by using an alignment rod, and avoidance of vascular structures along the trajectory route may be achieved by using CT angiography in conjunction with contrast-enhanced MRI. ${ }^{48}$

Overall, the strongest factor for minimizing complications is correct patient and target selection. This is a broad topic and involves all aspects of the procedure. This continues to be a moving target as we learn more about the capabilities and the limitations of the technology.

\section{Limitations}

This systematic review is not free of limitations. Due to the paucity of studies that directly compare LITT with 
other treatment modalities, the outcomes reported herein were derived mostly from retrospective studies, case series, and case reports. Of the 35 peer-reviewed articles from which we aggregated data, 19 were small case series or case reports. This is a rather large percentage, and it should be emphasized that there are inherent limitations of aggregated retrospective data. We have previously mentioned the heterogeneity in reporting that exists among the various series. We also acknowledge that varying degrees of bias against reporting poor outcomes or complications may exist, particularly in small retrospective case series and case reports. Although we cannot know the actual degree to which selective reporting affects the overall robustness of the data, we nonetheless recognize that it is a limitation of our paper and should be kept in mind when drawing conclusions. Furthermore, although LITT is indicated for a variety of pathologies, our analysis was limited only to a handful of pathologies that were addressed in the eligible articles. We originally attempted to describe the efficacy of LITT within the aggregate patient sample; however, this was precluded by the drastic heterogeneity between the reported outcomes. Future research requires large, randomized studies in which the safety and efficacy of LITT are compared to other treatment options, and longterm follow-up is required to further elucidate the impact of LITT on the natural course of different pathologies.

\section{Conclusions}

LITT is a minimally invasive procedure that shows promising outcomes in pediatric neurosurgery. The most common applications of LITT in pediatrics are treatment of medically refractory epilepsy and deep-seated brain tumors not amenable to resection. LITT is a safe option in pediatrics, and associated complications can be minimized through accurate treatment planning and proper ablation technique. Nevertheless, large prospective studies are needed to compare the efficacy and safety of LITT in pediatrics to those of other treatment modalities.

\section{References}

1. Ashraf O, Patel NV, Hanft S, Danish SF. Laser-induced thermal therapy in neuro-oncology: a review. World Neurosurg. 2018;112:166-177.

2. Ascher PW. Interstitial thermal therapy for brain tumors with Nd:YAG laser under real-time MRI control. In: Joffe SN, Atsumi K, eds. Laser Surgery: Advanced Characterization, Therapeutics, and Systems II. Vol 1200. International Society for Optics and Photonics; 1990.

3. Jethwa PR, Lee JH, Assina R, et al. Treatment of a supratentorial primitive neuroectodermal tumor using magnetic resonance-guided laser-induced thermal therapy. J Neurosurg Pediatr. 2011;8(5):468-475.

4. Kuo CH, Feroze AH, Poliachik SL, et al. Laser ablation therapy for pediatric patients with intracranial lesions in eloquent areas. World Neurosurg. 2019;121:e191-e199.

5. Dadey DY, Kamath AA, Leuthardt EC, Smyth MD. Laser interstitial thermal therapy for subependymal giant cell astrocytoma: technical case report. Neurosurg Focus. 2016;41(4):E9.

6. Tovar-Spinoza Z, Choi H. MRI-guided laser interstitial thermal therapy for the treatment of low-grade gliomas in children: a case-series review, description of the current technologies and perspectives. Childs Nerv Syst. 2016;32(10):1947-1956.
7. Tovar-Spinoza Z, Choi H. Magnetic resonance-guided laser interstitial thermal therapy: report of a series of pediatric brain tumors. J Neurosurg Pediatr. 2016;17(6):723-733.

8. Marcus J. Psychosocial issues in pediatric oncology. Ochsner J. 2012;12(3):211-215.

9. Fayed I, Sacino MF, Gaillard WD, et al. MR-guided laser interstitial thermal therapy for medically refractory lesional epilepsy in pediatric patients: experience and outcomes. $P e$ diatr Neurosurg. 2018;53(5):322-329.

10. Moher D, Liberati A, Tetzlaff J, Altman DG. Preferred reporting items for systematic reviews and meta-analyses: the PRISMA statement. PLoS Med. 2009;6(7):e1000097.

11. Kang JY, Wu C, Tracy J, et al. Laser interstitial thermal therapy for medically intractable mesial temporal lobe epilepsy. Epilepsia. 2016;57(2):325-334.

12. Boerwinkle VL, Foldes ST, Torrisi SJ, et al. Subcentimeter epilepsy surgery targets by resting state functional magnetic resonance imaging can improve outcomes in hypothalamic hamartoma. Epilepsia. 2018;59(12):2284-2295.

13. Buckley RT, Wang AC, Miller JW, et al. Stereotactic laser ablation for hypothalamic and deep intraventricular lesions. Neurosurg Focus. 2016;41(4):E10.

14. Cobourn K, Fayed I, Keating RF, Oluigbo CO. Early outcomes of stereoelectroencephalography followed by MR-guided laser interstitial thermal therapy: a paradigm for minimally invasive epilepsy surgery. Neurosurg Focus. 2018;45(3):E8.

15. Donos C, Breier J, Friedman E, et al. Laser ablation for mesial temporal lobe epilepsy: surgical and cognitive outcomes with and without mesial temporal sclerosis. Epilepsia. 2018; 59(7):1421-1432.

16. Du VX, Gandhi SV, Rekate HL, Mehta AD. Laser interstitial thermal therapy: a first line treatment for seizures due to hypothalamic hamartoma? Epilepsia. 2017;58(suppl 2):77-84.

17. Gadgil N, Lam S, Pan IW, et al. Staged magnetic resonanceguided laser interstitial thermal therapy for hypothalamic hamartoma: analysis of ablation volumes and morphological considerations. Neurosurgery. 2020;86(6):808-816.

18. Lewis EC, Weil AG, Duchowny M, et al. MR-guided laser interstitial thermal therapy for pediatric drug-resistant lesional epilepsy. Epilepsia. 2015;56(10):1590-1598.

19. Miller BA, Salehi A, Limbrick DD Jr, Smyth MD. Applications of a robotic stereotactic arm for pediatric epilepsy and neurooncology surgery. J Neurosurg Pediatr. 2017;20(4):364-370.

20. Schroeder JL, Missios S, Barnett GH, Mohammadi AM. Laser interstitial thermal therapy as a novel treatment modality for brain tumors in the thalamus and basal ganglia. Photonics Lasers Med. 2014;3(2):151-158.

21. Tovar-Spinoza Z, Ziechmann R, Zyck S. Single and staged laser interstitial thermal therapy ablation for cortical tubers causing refractory epilepsy in pediatric patients. Neurosurg Focus. 2018;45(3):E9.

22. Xu DS, Chen T, Hlubek RJ, et al. Magnetic resonance imaging-guided laser interstitial thermal therapy for the treatment of hypothalamic hamartomas: a retrospective review. Neurosurgery. 2018;83(6):1183-1192.

23. Feroze AH, McGrath M, Williams JR, et al. Laser interstitial thermal therapy for pediatric atypical teratoid/rhabdoid tumor: case report. Neurosurg Focus. 2020;48(1):E11.

24. Gonzalez-Martinez J, Vadera S, Mullin J, et al. Robot-assisted stereotactic laser ablation in medically intractable epilepsy: operative technique. Neurosurgery. 2014;10(2)(suppl 2):167-173.

25. Hooten KG, Werner K, Mikati MA, Muh CR. MRI-guided laser interstitial thermal therapy in an infant with tuberous sclerosis: technical case report. J Neurosurg Pediatr. 2018;23(1):92-97.

26. Karsy M, Patel DM, Bollo RJ. Trapped ventricle after laser ablation of a subependymal giant cell astrocytoma complicated by intraventricular gadolinium extravasation: case report. J Neurosurg Pediatr. 2018;21(5):523-527.

27. Tovar-Spinoza Z, Carter D, Ferrone D, et al. The use of MRI- 
guided laser-induced thermal ablation for epilepsy. Childs Nerv Syst. 2013;29(11):2089-2094.

28. Xu DS, Rosenfeld A, Ponce FA, et al. Cerebral peduncle tumor ablated by novel 3-mm laser tip. Stereotact Funct Neurosurg. 2015;93(1):38-41.

29. Riordan M, Tovar-Spinoza Z. Laser induced thermal therapy (LITT) for pediatric brain tumors: case-based review. Transl Pediatr. 2014:3(3):229-235.

30. Dadey DY, Kamath AA, Smyth MD, et al. Utilizing personalized stereotactic frames for laser interstitial thermal ablation of posterior fossa and mesiotemporal brain lesions: a singleinstitution series. Neurosurg Focus. 2016;41(4):E4.

31. Jethwa PR, Barrese JC, Gowda A, et al. Magnetic resonance thermometry-guided laser-induced thermal therapy for intracranial neoplasms: initial experience. Neurosurgery. 2012; 71(1)(Suppl Operative):133-145, 144-145.

32. Roux F, Merienne L, Leriche B, et al. Laser interstitial thermotherapy in stereotactical neurosurgery. Lasers Med Sci. 1992;7(1-4):121-126.

33. Southwell DG, Birk HS, Larson PS, et al. Laser ablative therapy of sessile hypothalamic hamartomas in children using interventional MRI: report of 5 cases. $J$ Neurosurg Pediatr. 2018;21(5):460-465.

34. Stellon MA, Cobourn K, Whitehead MT, et al. "Laser and the Tuber": thermal dynamic and volumetric factors influencing seizure outcomes in pediatric subjects with tuberous sclerosis undergoing stereoencephalography-directed laser ablation of tubers. Childs Nerv Syst. 2019;35(8):1333-1340.

35. Tandon V, Lang M, Chandra PS, et al. Is edema a matter of concern after laser ablation of epileptogenic focus? World Neurosurg. 2018;113:366-372.e3.

36. Wilfong AA, Curry DJ. Hypothalamic hamartomas: optimal approach to clinical evaluation and diagnosis. Epilepsia. 2013;54(suppl 9):109-114.

37. Sandoval-Garcia C, Karkare K, Miller I, et al. Long term outcomes of magnetic resonance (MR)-guided laser interstitial thermal therapy (MRgLITT) in pediatric epilepsy surgery. Paper presented at: 46th Annual Meeting of the International Society of Pediatric Neurosurgery; October 5-11, 2018; Tel Aviv, Israel.

38. Lopez Bon J, Handler M, Chapman K. Laser ablation: a new therapeutic option for children with pharmacoresistant lesional epilepsy. Paper presented at: 68th Annual Meeting of the America Epilepsy Society; December 5-9, 2014; Seattle, WA.

39. Missios S, Bekelis K, Barnett GH. Renaissance of laser interstitial thermal ablation. Neurosurg Focus. 2015;38(3):E13.

40. Voigt JD, Torchia M. Laser interstitial thermal therapy with and without MRI guidance for treatment of brain neoplasms - a systematic review of the literature. Photonics Lasers Med. 2014;3(2):77-93.

41. Banerjee C, Snelling B, Berger MH, et al. The role of magnetic resonance-guided laser ablation in neurooncology. $\mathrm{Br} J$ Neurosurg. 2015;29(2):192-196.

42. Buttrick S, Komotar RJ. Introduction for laser interstitial thermal therapy (LITT) in neurosurgery supplement. Neurosurgery. 2016;79(suppl 1):S1-S2.

43. Sharma M, Ugiliweneza B, Wang D, et al. National trends and factors predicting outcomes following laser interstitial thermal therapy for brain lesions: a nationwide inpatient sample analysis. World Neurosurg. 2020;139:e88-e97.

44. Patel P, Patel NV, Danish SF. Intracranial MR-guided laserinduced thermal therapy: single-center experience with the Visualase thermal therapy system. J Neurosurg. 2016;125(4): 853-860.

45. Wu C, Jermakowicz WJ, Chakravorti S, et al. Effects of surgical targeting in laser interstitial thermal therapy for mesial temporal lobe epilepsy: a multicenter study of 234 patients. Epilepsia. 2019;60(6):1171-1183.

46. Régis J, Scavarda D, Tamura M, et al. Gamma knife surgery for epilepsy related to hypothalamic hamartomas. Semin Pediatr Neurol. 2007;14(2):73-79.

47. Pati S, Sollman M, Fife TD, Ng YT. Diagnosis and management of epilepsy associated with hypothalamic hamartoma: an evidence-based systematic review. J Child Neurol. 2013; 28(7):909-916.

48. Pruitt R, Gamble A, Black K, et al. Complication avoidance in laser interstitial thermal therapy: lessons learned. J Neurosurg. 2017;126(4):1238-1245.

49. Holste KG, Orringer DA. Laser interstitial thermal therapy. Neurooncol Adv. 2019;2(1):vdz035.

50. Kameyama S, Shirozu H, Masuda H, et al. MRI-guided stereotactic radiofrequency thermocoagulation for 100 hypothalamic hamartomas. J Neurosurg. 2016;124(5):1503-1512.

51. Hader WJ, Tellez-Zenteno J, Metcalfe A, et al. Complications of epilepsy surgery: a systematic review of focal surgical resections and invasive EEG monitoring. Epilepsia. 2013;54(5):840-847.

52. Kim SK, Wang KC, Hwang YS, et al. Epilepsy surgery in children: outcomes and complications. J Neurosurg Pediatr. 2008;1(4):277-283.

53. Ng YT, Rekate HL. Endoscopic resection of hypothalamic hamartoma for refractory epilepsy: preliminary report. Semin Pediatr Neurol. 2007;14(2):99-105.

54. Grewal SS, Zimmerman RS, Worrell G, et al. Laser ablation for mesial temporal epilepsy: a multi-site, single institutional series. J Neurosurg. 2019;130(6):2055-2062.

55. Régis J, Bartolomei F, de Toffol B, et al. Gamma knife surgery for epilepsy related to hypothalamic hamartomas. Neurosurgery. 2000;47(6):1343-1352.

56. Barbaro NM, Quigg M, Ward MM, et al. Radiosurgery versus open surgery for mesial temporal lobe epilepsy: The randomized, controlled ROSE trial. Epilepsia. 2018;59(6):1198-1207.

57. Lassen B, Helseth E, Egge A, et al. Surgical mortality and selected complications in 273 consecutive craniotomies for intracranial tumors in pediatric patients. Neurosurgery. 2012; 70(4):936-943.

58. Neervoort FW, Van Ouwerkerk WJ, Folkersma H, et al. Surgical morbidity and mortality of pediatric brain tumors: a single center audit. Childs Nerv Syst. 2010;26(11):1583-1592.

59. Hodgson DC, Goumnerova LC, Loeffler JS, et al. Radiosurgery in the management of pediatric brain tumors. Int $J$ Radiat Oncol Biol Phys. 2001;50(4):929-935.

60. Lo SS, Fakiris AJ, Abdulrahman R, et al. Role of stereotactic radiosurgery and fractionated stereotactic radiotherapy in pediatric brain tumors. Expert Rev Neurother. 2008;8(1):121-132.

61. Salem U, Kumar VA, Madewell JE, et al. Neurosurgical applications of MRI guided laser interstitial thermal therapy (LITT). Cancer Imaging. 2019;19(1):65.

\section{Disclosures}

Dr. Danish received honoraria from Medtronic.

\section{Author Contributions}

Conception and design: Danish, Zeller, Kaye, Jumah. Acquisition of data: Zeller, Kaye, Mantri, Mir. Analysis and interpretation of data: Danish, Zeller, Kaye, Jumah, Raju. Drafting the article: Zeller, Kaye, Mantri, Mir. Critically revising the article: Danish, Zeller, Kaye, Jumah. Reviewed submitted version of manuscript: all authors. Approved the final version of the manuscript on behalf of all authors: Danish. Statistical analysis: Zeller, Jumah. Administrative/technical/material support: Danish, Raju. Study supervision: Jumah.

\section{Correspondence}

Shabbar F. Danish: Rutgers-RWJ Medical School, New Brunswick, NJ.danishsh@cinj.rutgers.edu. 